Fen Bilimleri Dergisi

\title{
METHOD OF SEPARATION OF VARIABLES
}

\author{
Mehmet KARAKAS \\ Sakarya University Vocational School of Sakarya \\ Sakarya/TURKEY \\ E-mail:mkarakas@sakarya.edu.tr
}

\begin{abstract}
Throughout this article I've been talking about and solving partial diferential equations, using the method of separition of variable.Verify that the partial differential equation is linear and homogeneous.One of the ordinary differential equations will be a boundary value problem determining the eigenvalues and eigenfunctions fort the problem.
\end{abstract}

\section{Introduction}

We intend to teach the physical principles of the heat equation and its initial and boundary conditions, to pursue the mathematical solution of some typical problems involving partial differential equations. We will use a technique called the separation of variables. We will discuss a few examples, emphasizing problem solving techniques, but realizing how not to misuse the technique.[1], [2]

A relatively simple, but typical, problem for the equation of heat conduction occurs for aone-dimensional rod $(0 \leq x \leq L)$ when all the thermal coefficients are constant. Then the PDE,

$$
\frac{\partial u}{\partial t}=k \frac{\partial^{2} u}{\partial x^{2}}+\frac{Q(x, t)}{c p} \quad \begin{array}{ll}
t>0 \\
0<x<L
\end{array}
$$

must be solved subject to the initial condition,

$$
u(x, 0)=f(x) \quad 0<x<L
$$


and two boundary conditions. For example, if both ends of the rod have prescribed temperature, then

$$
\begin{aligned}
& u(0, t)=T_{1}(t) \\
& u(L, t)=T_{2}(t)
\end{aligned} \quad t>0
$$

The method of separation of variables is used when the partial differential equation and the boundary conditions are linear and homogeneous, concepts we now explain. [3]

\section{Linearity}

As in the study of ordinary differential equations, the concept of linearity will be very important for us. A linear operator $L$ by definition satisfies

$$
L\left(c_{1} u_{1}+c_{2} u_{2}\right)=c_{1} L\left(u_{1}\right)+c_{2} L\left(u_{2}\right)
$$

for any two functions $u_{1}$ and $u_{2}$, where $c_{1}$ and $c_{2}$ are arbitrary constants. $\partial / \partial t$ and $\partial^{2} / \partial x^{2}$ are examples of linear aperators since they satisfy $(2.2 .1)$ :

$$
\begin{aligned}
& \frac{\partial}{\partial t}\left(c_{1} u_{1}+c_{2} u_{2}\right)=c_{1} \frac{\partial u_{1}}{\partial t}+c_{2} \frac{\partial u_{2}}{\partial t} \\
& \frac{\partial^{2}}{\partial x^{2}}+\left(c_{1} u_{1}+c_{2} u_{2}\right)=c_{1} \frac{\partial^{2} u_{1}}{\partial x^{2}}+c_{2} \frac{\partial^{2} u_{2}}{\partial x^{2}}
\end{aligned}
$$

It can be shown that any linear combination of linear operators is a linear operator. Thus, the heat operator

$$
\frac{\partial}{\partial x^{2}}-k \frac{\partial^{2}}{\partial x^{2}}
$$

is also a linear operator. A linear equation for $u$ is of the form

$$
L(u)=f
$$

where $L$ is a linear operator and $f$ is known. Examples of linear partial differential equations are [4] 


$$
\begin{aligned}
& \frac{\partial u}{\partial t}=k \frac{\partial^{2} u}{\partial x^{2}}+f(x, t) \\
& \frac{\partial u}{\partial t}=k \frac{\partial^{2} u}{\partial x^{2}}+a(x, t) u+f(x, t) \\
& \frac{\partial^{2} u}{\partial x^{2}}+\frac{\partial^{2} u}{\partial y^{2}}=0 \\
& \frac{\partial u}{\partial t}=\frac{\partial^{3} u}{\partial x^{3}}+a(x, t) u
\end{aligned}
$$

Examples of nonlinear partial differential equations are

$$
\begin{aligned}
& \frac{\partial u}{\partial t}=k \frac{\partial^{2} u}{\partial x^{2}}+a(x, t) u^{4} \\
& \frac{\partial u}{\partial t}+u \frac{\partial u}{\partial x}=\frac{\partial^{3} u}{\partial x^{3}}
\end{aligned}
$$

That $u^{4}$ and $u \partial u / \partial x$ terms are nonlinear; they do not satisfy (2.1).

If $f=0$, then becomes $L(u)=0$, called a linear homogeneous equation. Examples of linear homogeneous partial differential equations include the heat equation,

$$
\frac{\partial u}{\partial t}-k \frac{\partial^{2} u}{\partial x^{2}}=0
$$

as well as $(2.3 c)$ and $(2.3 d)$. From $(2.1)$ it follows that $L(0)=0\left(\right.$ let $\left.c_{1}=0 \quad c_{2}=0\right)$. Therefore, $u=0$ is always a solution of a linear homogeneous equation. For example, $u=0$ satisfies the heat equation (2.4). We call $u=0$ the trivial solution of a linear homogeneous equation. The simplest way to test whether an equation is homogeneous is to substitute the function $u$ identically equal to zero. If $u \equiv 0$ satisfies a linear equation, then is must be that $f=0$ and hence the linear equation is homogeneous. Otherwise, the equation is said to be nonhomogeneous [e.g. , (2.3a) and $(2.3 b)$ ]. 
The fundamental property of linear operators (2.1) allows solutions of linear equations to be added below:

\section{Principle of Superposition}

If $u_{1}$ and $u_{2}$ satisfy a linear homogeneous equation, then an arbitrary linear combination of them, $c_{1} u_{1}+c_{2} u_{2}$, also satisfies the same linear homogeneous equation.

This proof depends on the definition of a linear operator. Suppose that $u_{1}$ and $u_{2}$ are two solutions of a linear homogeneous equation. That means that $L\left(u_{1}\right)=0$ and $L\left(u_{2}\right)=0$. Let us calculate $L\left(c_{1} u_{1}+c_{2} u_{2}\right)$. From the definition of a linear operator,

$$
L\left(c_{1} u_{1}+c_{2} u_{2}\right)=c_{1} L\left(u_{1}\right)+c_{2} L\left(u_{2}\right)
$$

Since $u_{1}$ and $u_{2}$ are homogeneous solutions, it follows that $L\left(c_{1} u_{1}+c_{2} u_{2}\right)=0$. This means that $c_{1} u_{1}+c_{2} u_{2}$ satisfies the linear homogeneous equation $L(u)=0$ if $u_{1}$ and $u_{2}$ satisfy the same linear homogeneous equation. [5]

The concepts of linearity and homogeneity also apply to boundary conditions, here variables are evaluated at specific points. Examples of linear boundary conditions are the conditions which we have discussed:

$$
\begin{aligned}
& u(0, t)=f(t) \\
& \frac{\partial u}{\partial x}(L, t)=g(t) \\
& \frac{\partial u}{\partial x}(0, t)=0 \\
& -K_{0} \frac{\partial u}{\partial x}(L, t)=h[u(L, t)-g(t)]
\end{aligned}
$$

A nonlinear boundary condition, for example, would be

$$
\frac{\partial u}{\partial x}(L, t)=u^{2}(L, t)
$$


Only $(2.5 c)$ is satisfied by $u \equiv 0$ (of the linear conditions) and hence is homogeneous. It is not necessary that a boundary condition be $u(0, t)=0$ for $u \equiv 0$ to satisfy it.

\section{HEAT EQUATION WITH ZERO TEMPERATURES AT FINITE ENDS \\ Introduction}

Partial differential equation (2.1) is linear, but it is homogeneous only if there are no sources, $Q(x, t)=0$. The boundary conditions (2.3) are also linear, and they too are homogeneous only if $T_{1}(t)=0$ and $T_{2}(t)=0$. We thus first propose to study

$$
\begin{array}{lll}
\text { PD: } & \frac{\partial u}{\partial t}=k \frac{\partial^{2} u}{\partial x^{2}} & 0<x<L \\
\text { BC: } & u(0, t)=0 & \\
& u(L, t)=0 & \\
\text { IC: } & u(x, 0)=f(x) &
\end{array}
$$

The problem consists of a linear homogeneous partial differential equation with linear homogeneous boundary conditions. There are two reasons for our investigating this type of problem, (3.1)-(3.3), then we claim it can be solved by the method of separation of variables. First, this problem is a relevant physical problem corresponding to a one-dimensional rod $(0<x)[6],[7]$

\section{APPENDIX: ORTHOGONALITY OF FUNCTIONS}

Two vectors $\mathbf{A}$ and $\mathbf{B}$ are orthogonal if $A . B=0$. In component form: $A=a_{1} \hat{i}+a_{2} \hat{j}+a_{3} \hat{k}$ and $B=b_{1} \hat{i}+b_{2} \hat{j}+b_{3} \hat{k} ; \mathbf{A}$ and $\mathbf{B}$ are orthogonal if $\sum_{i} a_{i} b_{i}=0$. A function $A(x)$ can be thought of as a vector. If only three values of $x$ are important, $x_{1}, x_{2}$, and $x_{3}$, then the components of the function $A(x)$ (thought of as a vector) are $A\left(x_{1}\right) \equiv a_{1}, A\left(x_{2}\right) \equiv a_{2}$, and $A\left(x_{3}\right) \equiv a_{3}$. The function $A(x)$ is orthogonal to the 
function $B(x)$ (by definition) if $\sum_{i} a_{i} b_{i}=0$. However, in our problems, all values of $x$ between 0 and $L$ are important. The function $A(x)$ can be thought of as an infinitedimensional vectori whose components are $A\left(x_{i}\right)$ for all $x_{i}$ on some interval. In this manner the function $A(x)$ would be said to be orthogonal to $B(x)$ if $\sum_{i} A\left(x_{i}\right) B\left(x_{i}\right)=0$, where the summation was to include all points between 0 and $L$.It is thus natural to define the function $A(x)$ to be orthogonal to $B(x)$ if $\int_{0}^{L} A(x) B(x) d x=0$

The integral replaces the vector dot product; bot hare examples of "inner products."

In vectors, we have the three mutually perpendicular (orthogonal) unit vectors $\hat{i}, \hat{j}$ and $\hat{k}$ known as the standard basis vectors. In somponent form

$$
A=a_{1} \hat{i}+a_{2} \hat{j}+a_{3} \hat{k}
$$

$a_{1}$ is the projection of $\mathbf{A}$ in the $\hat{i}$ direction, and so on. Sometimes we wish to represent $\mathbf{A}$ in terms of other mutually orthogonal vectors (which may not be unit vectors) $\mathbf{u}, \mathbf{v}$, and $\mathbf{w}$, called an orthogonal set of vectors. Then

$$
A=a_{u} u+a_{v} v+a_{w} w
$$

To determine the coordinates $a_{u}, a_{v}, a_{w}$ with respect to this orthogonal set, $\mathbf{u}$, $\mathbf{v}$, and $\mathbf{w}$, we can form certain dot products. For example,

$$
A \cdot u=a_{u} u \cdot u+a_{v} \cdot u+a_{w} w \cdot u
$$

Note that $v \cdot u=0$ and $w \cdot u=0$, since we assumed that new set was mutually orthogonal. Thus, we can easily solve for the coordinate $a_{u}$ of $\mathbf{A}$ in the $\mathbf{u}$ direktion,

$$
a_{u}=\frac{A . u}{u . u}
$$

( $a_{u} u$ is the vector projection of $\mathbf{A}$ in the $\mathbf{u}$ direction.) 
For functions, we can do a similar thing. If $f(x)$ can be represented by alinear combination of the orthogonal set, $\sin n \pi x / L$, then

$$
f(x)=\sum_{n=1}^{\infty} B_{n} \sin \frac{n \pi x}{L}
$$

where the $B_{n}$ may be interpreted as the coordinates of $f(x)$ with respect to the "direction" (or basis vector) $\sin n \pi x / L$. To determine these coordinates we take the inner product with an arbitrary basis function (vector) $\sin m \pi x / L \mathrm{i}$ where the inner product of two functions is the integral of their product. Thus, as before, [9],[12]

$$
\begin{aligned}
& \int_{0}^{L} f(x) \sin \frac{m \pi x}{L} d x=\sum_{n=1}^{\infty} B_{n} \int_{0}^{L} \sin \frac{n \pi x}{L} \sin \frac{m \pi x}{L} d x \\
& \text { Since } \sin m \pi x / L \text { is an orthogonal set of functions, } \\
& \int_{0}^{L} \sin n \pi x / L \sin m \pi x / L d x=0 \text { for } n \neq m \text {. Hence, we solve fort he coordinate } \\
& \text { (coefficient) } B_{n}: \\
& B_{n}=\frac{\int_{0}^{L} f(x) \sin n \pi x / L d x}{\int_{0}^{L} \sin ^{2} n \pi x / L d x}
\end{aligned}
$$

This is seen to be the same idea as the projection Formula (4.2).

Our Standard formula $(2.3 .31 b)$,

$$
\begin{aligned}
& \int_{0}^{L} \sin ^{2} n \pi x / L d x=L / 2, \text { return (2.3.A3) to the more familiar form, } \\
& B_{n}=\frac{2}{L} \int_{0}^{L} f(x) \sin \frac{n \pi x}{L} d x
\end{aligned}
$$

Both formulas (4.2) and (4.3) are divided by something. In (4.2) it is $u \cdot u$, or the length of the vector $\mathbf{u}$ squared. Thus, $\int_{0}^{L} \sin ^{2} n \pi x / L d x$ may be thought of as the 
length squared of $\sin n \pi x / L$ (althougd here length means nothing other than the square root of the integral). In this manner the length squared of the function $\sin n \pi x / L$ is $L / 2 \mathrm{i}$ which is an explanation of the appearence of the term $2 / L$ in $(4.4)$.

\section{WORKED EXAMPLES WITH THE HEAT EQUATION (OTHER BOUNDARY VALUE PROBLEMS)}

\section{Heat Conduction in a Rod with Insulated Ends}

Let us work out in detail the solution (and its interpretation ) of the following problem defined for $0 \leq x \leq L$ and $t \geq 0$ :

$$
\begin{array}{ll}
\text { PDE: } \quad & \frac{\partial u}{\partial t}=k \frac{\partial^{2} u}{\partial x^{2}} \\
\text { BC: } \quad & \frac{\partial u}{\partial x}(0, t)=0 \\
\frac{\partial u}{\partial t}(L, t)=0 & \\
\text { IC: } \quad u(x, 0)=f(x)
\end{array}
$$

As a review, this is a heat conduction problem in a one-dimensional rod with constant thermal properties and no sources. This problem is quite similar to the problem indicated in the boundary conditions. Here the result, but the ends were fixed at $0^{\circ}$. Both the partial differential equation and the boundary conditions are linear and homogeneous. Consequently, we apply the method of separation ofvariables. We may follow the general procedure described in The assumption of product solutions,

$$
u(x, t)=\phi(x) G(t)
$$

implies from the PDE as before that

$$
\begin{aligned}
& \frac{d G}{d t}=-\lambda k G \\
& \frac{d^{2} \phi}{d x^{2}}=-\lambda \phi
\end{aligned}
$$


where $\lambda$ is the separation constant. Again,

$$
G(t)=c e^{-\lambda k t}
$$

The insulated boundary conditions, $(5.1 b)$ and $(5.1 c)$, imply that the separated solutions must satisfy $d \phi / d x(0)=0$ and $d \phi / d x(L)=0$. The separation constant $\lambda$ is then determined by finding those $\lambda$ for which nontrivial solutions exist fort he following boundary value problem:

$$
\begin{aligned}
& \frac{d^{2} \phi}{d x^{2}}=-\lambda \phi \\
& \frac{d \phi}{d x}(0)=0 \\
& \frac{d \phi}{d x}(L)=0 .
\end{aligned}
$$

Although the ordinary differential equation fort he boundary value problem is the same one as previously analyzed, the boundary conditions are different.

\section{APPENDIX: MATRIX EIGENVALUE PROBLEM AND}

\section{ORTHOGONALITY OF EIGENVECTORS}

The matrix eigenvalue problem

$$
A x=\lambda x
$$

where $\mathrm{A}$ is an $n \times n$ real matrix (with entries $a_{i j}$ ) and $\mathrm{x}$ is an $n$-dimensional column vector (with components $x_{i}$ ), has many properties similar to those of the SturmLiouville eigenvalue problem.

Eigenvalues and eigenvectors. For all values of $\lambda, x=0$ is a "trivial" solution of the homogeneous linear system (6.1). We ask, for what values of $\lambda$ are there nontrivial solutions? In general, (6.1) can be rewritten as

$$
(A-\lambda \mathrm{I}) x=0
$$


where $\mathbf{I}$ is the identity matrix. According to the theory of linear equations (elementary linear algebra), a nontrivial solution exists only if

$$
\operatorname{det}[A-\lambda \mathrm{I}]=0 \text {. }
$$

Such values of $\lambda$ are called eigenvalues, and the corresponding nonzero vectors $\mathbf{x}$ called eigenvectors.

In general, (6.3) yields an $n$ th-degree polynomial (known as the characteristic polynomial) - determines the eigenvalues; there will be $n$ eigenvalues (but they may not be distinct). Corresponding to each distinct eigenvalue, there will be an eigenvector. [14]

Example. If A $A=\left[\begin{array}{ll}2 & 1 \\ 6 & 1\end{array}\right]$, then the eigenvalues satisfy

$$
0=\operatorname{det}\left[\begin{array}{cc}
2-\lambda & 1 \\
6 & 1-\lambda
\end{array}\right]=(2-\lambda)(1-\lambda)-6=\lambda^{2}-3 \lambda-4=(\lambda-4)(\lambda+1)
$$

The characteristic polynomial. The eigenvalues are $\lambda=4$ and $\lambda=-1$. For $\lambda=4,(6.1)$ becomes

$2 x_{1}+x_{2}=4 x_{1}$ and $6 x_{1}+x_{2}=4 x_{2}$ or equivalently $x_{2}=2 x_{1}$. The eigenvector $\left[\begin{array}{l}x_{1} \\ x_{2}\end{array}\right]=x_{1}\left[\begin{array}{l}1 \\ 2\end{array}\right]$ is an arbitrary multiple of $\left[\begin{array}{l}1 \\ 2\end{array}\right]$ for $\lambda=4$. For $\lambda=-1$

$$
2 x_{1}+x_{2}=-x_{1} \text { and } 6 x_{1}+x_{2}=-x_{2} \text { and thus the eigenvector }\left[\begin{array}{l}
x_{1} \\
x_{2}
\end{array}\right]=x_{1}\left[\begin{array}{c}
1 \\
-3
\end{array}\right] \text { is }
$$
an arbitrary multiple of $\left[\begin{array}{c}1 \\ -3\end{array}\right]$.

Green's Formula. The matrix A may be thought of as a linear operator in the same way that

$$
L=\frac{d}{d x}\left(p \frac{d}{d x}\right)+q \text { is a linear differential operator. A operates on } n \text {-dimensional }
$$
vectors producing an $n$-dimensional vector, while $L$ operates on functions and yields a 
function. In analyzing the Sturm-Liouville eigenvalue problem, orm's Formula was important:

$$
\int_{a}^{b}[u L(v)-v L(u)] d x=p\left(u \frac{d v}{d x}-v \frac{d u}{d x}\right) \mathrm{I}_{b}^{a}
$$

where $u$ and $v$ are arbitrary functions. Often, the boundary terms vanished. For vectors, the dot product is analogous to integration, $\mathrm{a} \cdot \mathrm{b}=\sum_{i} a_{i} b_{i}$, where $a_{i}$ and $b_{i}$ are the $i$ th components of, respectively, a and $\mathbf{b}$ (see Sec.2. 3 Appendix). By direct analogy to orm's Formula we would be lead to investigate $\mathbf{u} . \mathbf{A v}$ and $\mathbf{v} . \mathbf{A u}$, where $\mathbf{u}$ and $\mathbf{v}$ are arbitrary vectors.

Instead, we analyze u.Av and v.Au, where $\mathbf{B}$ is any $n \times n$ matrix:

$$
\begin{aligned}
& \mathbf{u . A v}=\sum_{i}\left(u_{i} \sum_{j} a_{i j} v_{j}\right)=\sum_{i} \sum_{j} a_{i j} u_{i} v_{j} \\
& \mathbf{v} . \mathbf{B u}=\sum_{i}\left(v_{i} \sum_{j} b_{i j} u_{j}\right)=\sum_{i} \sum_{j} b_{i j} u_{i} v_{j}=\sum_{i} \sum_{j} b_{i j} u_{i} v_{j},
\end{aligned}
$$

where an alternative expression for v.Bu was derived by interchanging the roles of $i$ and $j$. Thus,

$$
\text { u. } \mathrm{Av}-\mathrm{v} \cdot \mathrm{Bu}=\sum_{\mathrm{i}} \sum_{\mathrm{j}}\left(a_{i j}-b_{j i}\right) u_{i} v_{j},
$$

If formul B equal the transpose of $\mathbf{A}\left(i . e ., b_{j i}=a_{i j}\right)$, whose notation is $B=A^{t}$, then we have the following theorem:

$$
u . A v-v \cdot A^{t} u=0,
$$

quite analogous to form's formula.

Self-adjointness. The difference between $\mathbf{A}$ and its transposei $A^{t}$, in (6.4) causes insurmountable difficulties for us. We will thus restrict our attention to symmetric matrices, in which case $A=A^{t}$. For symmetric matrices

$$
u . A v-v . A u=0
$$


and we will be able to use this result to prove the same theorems about eigenvalues and eigenvectors for matrices as we proved about Sturm-Liouville eigenvalue problems.

For symmetric martrices, eigenvectors corresponding to different eigenvalues are orthogonal. To prove this, suppose that $\mathbf{u}$ and $\mathbf{v}$ are eigenvectors corresponding to $\lambda_{1}$ and $\lambda_{2}$, respectively:

$$
A u=\lambda_{1} u \text { and } A v=\lambda_{2} v
$$

If we directly apply $(6.5)$, then

$$
\left(\lambda_{2}-\lambda_{1}\right) u \cdot v=0
$$

Thus, if $\lambda_{1} \neq \lambda_{2}$ (different eigenvalues), the corresponding eigenfunctions are orthogonal in the sense that

$$
u . v=0 .
$$

We leave as an exercise the prof that the eigenvalues of a symmetric matrix are real.

Example. The eigenvalues of the real symmetric matrix $\left[\begin{array}{ll}6 & 2 \\ 2 & 3\end{array}\right]$ are determined from $(6-\lambda)(3-\lambda)-4=\lambda^{2}-9 \lambda+14=(\lambda-7)(\lambda-2)=0$.

For $\lambda=2$, the eigenvector satisfies

$$
6 x_{1}+2 x_{2}=2 x_{1} \text { and } 2 x_{1}+3 x_{2}=2 x_{2} \text {, }
$$

and hence $\left[\begin{array}{l}x_{1} \\ x_{2}\end{array}\right]=x_{1}\left[\begin{array}{c}1 \\ -2\end{array}\right]$. For $\lambda=7$, it follows that

$$
6 x_{1}+2 x_{2}=7 x_{1} \text { and } 2 x_{1}+3 x_{2}=7 x_{2},
$$

and the eigenvector is $\left[\begin{array}{l}x_{1} \\ x_{2}\end{array}\right]=x_{2}\left[\begin{array}{l}2 \\ 1\end{array}\right]$. As we have just proved for any real symmetric matrix, the eigenvectors are orthogonal, $\left[\begin{array}{c}1 \\ -2\end{array}\right] \cdot\left[\begin{array}{l}2 \\ 1\end{array}\right]=2-2=0$.

Eigenvector expansions. For real symmetric matrices it can be shown that if an eigenvalue repeats $R$ times, there will be $R$ independent eigenvectors zorresponding 
to that eigenvalue. These eigenvectors are automatically orthogonal to any eigenvectrs corresponding to a diferent eigenvalue. The Gram-Schmidt procedure can be applied so that all $R$ eigenvectors zorresponding to the same eigenvalue can be constructed to be mutually orthogonal. In this manner, for real symmetric $n \times n$ matrices, $n$ orthogonal eigenvectors can always be obtained. Since these vectors are orthogonal, they span the $n$-dimensional vector space and may be chosen as basis vectors. Any vector $\mathbf{v}$ may be represented in a series of the eigenvectors:

$$
v=\sum_{i=1}^{n} c_{i} \phi_{i}
$$

where $\phi_{i}$ is the $i$ th eigenvector. For regular Sturm-Liouville eigenvalue problems, the eigenfunctions are complete, meaning that any (piecewise smooth) function can be represented in terms of an eigenfunction expansion

$$
f(x) \approx \sum_{i=1}^{\infty} c_{i} \phi_{i}(x) .
$$

This is analogous to (6.7). In (6.8) the Fourier coefficients $c_{i}$ are determined by the orthogonality of the eigenfunctions. Similarly, the coordinates $c_{i}$ in (6.7) are determined by the orthogonality of the eigenvectors. We dot equation (6.7) into

$$
v \cdot \phi_{m}=\sum_{i=1}^{n} c_{i} \phi_{i} \cdot \phi_{m}=c_{m} \phi_{m} . \phi_{m}
$$

Since $\phi_{i} \cdot \phi_{m}=0, i \neq m$, determining $c_{m}$.

Linear systems. Sturm-Liouville eigenvalue problems are arise in separating variables for partial differential equations. In any way the matrix eigenvalue problem occurs is in "separating" a linear homogeneous system of ordinary differential equations with constant coefficients. A linear homogeneous first-order system of differential equations may be represented by

$$
\frac{d v}{d t}=A v
$$


where $\mathbf{A}$ is an $n \times n$ matrix and $\mathbf{v}$ is the desired $n$-dimensional vector solution. $\mathbf{v}$ usually satisfies given initial conditions, $v(0)=v_{0}$. We seek special solutions of the form of simple exponentials:

$$
v(t)=e^{\lambda t} \phi
$$

where $\phi$ is a constant vector. This is analogous to seeking product solutions by the method of separation of variables. Since $d v / d t=\lambda e^{\lambda t} \phi$, it follows that

$$
A \phi=\lambda \phi
$$

Thus, there exist solutions to (6.9) of the form (6.10) if $\lambda$ is an eigenvalue of A and $\phi$ is a corresponding eigenvector. We now restrict our attention to real symmetric matrices $\mathbf{A}$. There will always be $n$ mutually orthogonal eigenvectors $\phi_{i}$. We have obtained $n$ special solutions to the linear homogeneous system (6.9). A principle of superposition exists, and hence a linear combination of these solutions also satisfies (6.9):

$$
v=\sum_{i=1}^{n} c_{i} e^{\lambda i t} \phi_{i}
$$

We attempt to determine $c_{i}$ so that (6.12) satisfies the initial conditions, $v(0)=v_{0}$ :

$$
v_{0}=\sum_{i=1}^{n} c_{i} \phi_{i}
$$

Here, the orthogonality of the eigenvectors is helpful, and thus, as before,

$$
c_{i}=\frac{v_{0} \cdot \phi_{i}}{\phi_{i} \cdot \phi_{i}}
$$




\section{References}

[1]. Abramowitz, M., and Stegun, I.A., eds Handbook of Mathematical Functions.New York:Dover, 1965.

[2]. Antman, S.S., "The equations for lange Vibrations of Strings. "Awer, Math.mounthly 87,88,359-370. 1980

[3]. Bender, C.M., and Orszag, S.A. Advanced Mathematical Methods for Scientists and Engineers, New York: MCgrawltil, 1978

[4]. Berg, P.W., and Mcgregor, J.L. Elementary pential Differential Equations. San Francisco:Holden-Day 1966

[5]. Boyce, W.E., and Prima DI, R.C. Elementary Differential Equations and Boundary Value Problems.New York: Wiley 1977

[6]. Churchill, R.V., and Brown, J.W. Fourier Series and Bounddary Value Problems 3rd ed. New York: MCGrawnltil, 1978

[7]. Courant, R., and Hicbert, D. Methodsds of mathematical Physics, Vols.Lond2 New York:Wiley, 1953

[8]. Duff, G.F.D., and Haylor, D. Differantial Equations of Applied mathematics.New York: Wiley, 1966

[9]. Haberman, R. Mathematical Models:Mechanical Vibrations, Population Dynamics, and Traffic Flow.Englewood Cliffs, N.J.: Prentice-Hall 1977

[10]. Hildebrand, F.B. Advanced Calculus forApplications.2nd ed.Englewood Cliffs, N.J.: Prentice-Hall, 1976

[11]. Isaacson, E., and Keller, H.B.Analysis of numerical methods.New York:Wiley, 1981.

[12]. Sagan, H. Boundany and Eigen value Problems in Mathematical Physics. New York: Willey, 1961.

[13]. Smith, G.D. Numerical Solwdion Of Partial Differantial. New York:Oxford University press, 1915

[14]. Strang,G. Linnar Alpebre and its Applications, 2nd ed. New York: Academic Press, 1980

[15]. Young, E.C. Partral Differantial Equations Bostoy:Allyy and Bacon, 1972

[16]. Zauderer, E. Partial Differautial Equatiouns of Appliet mathematics. New York:Wiley, 1983. 